\section{Insecticidal activity of imidacloprid derivatives with an alkoxy group at the C5 position of the pyridine ring}

\author{
Shinzo Kagabu, * Yuya FuJII and \\ Keiichiro NISHIMURA ${ }^{\dagger}$ \\ Department of Chemistry, Faculty of Education, \\ Gifu University, Gifu 501-1193, Japan \\ ${ }^{\dagger}$ Research Institute for Advanced Science and Technology, \\ Osaka Prefecture University, Osaka 599-8570, Japan
}

(Received August 26, 2005; Accepted December 1, 2005)

Six derivatives of imidacloprid with an alkoxy group introduced at the fifth position on the pyridine ring were prepared. Minimal lethal doses in mol (MLDs) were determined in American cockroaches both with and without synergists, piperonyl butoxide and propargyl propyl benzenephosphonate. The $\log (1 / \mathrm{MLD})$ value without synergists was 7.43 for the methoxy substituted derivative. Values for the higher alkyloxy homologues particularly the $n$-propoxy derivative were lower. Synergists combined enhanced the potency by about one log unit for each compound. The introduction of any alkoxy group does not improve the activity of imidacloprid. (C) Pesticide Science Society of Japan

Keywords: neonicotinoid insecticide, imidacloprid, American cockroach, insecticidal activity, alkoxy-substituted pyridine.

\section{Introduction}

The preceding paper presented the insecticidal activity of halogen, cyano, and nitro-substituted imidacloprid at the 5 th position on the pyridine ring, and described that fluoro and chloro derivatives (Fig. 1) have strong insecticidal activity toward American cockroaches. ${ }^{1)}$ The result was unexpected, because an extra sub-<smiles>[X]c1cc(CN2CCNC2=[N+]([O-])[O-])cnc1Cl</smiles>
1a: imidacloprid $(\mathrm{X}=\mathrm{H})$
1b: 5-fluoroimidacloprid $(X=F)$
1c: 5-chloroimidacloprid $(\mathrm{X}=\mathrm{Cl})$

Fig. 1. Imidacloprid and 5-fluoro and 5-chloro derivatives.

\footnotetext{
* To whom correspondence should be addressed.

E-mail:kagabus@cc.gifu-u.ac.jp

(C) Pesticide Science Society of Japan
}

stitution, regardless of the nature or position of the substituent, had been assumed to reduce the potency of imidacloprid. ${ }^{2)}$ We were curious about how other substituents affect the insecticidal activity. In this note we describe the insecticidal activity of six alkoxy-substituted derivatives of imidacloprid.

\section{Materials and Methods}

\section{Preparation of compounds}

All melting points (mp) are uncorrected. NMR spectra were obtained with a Varian Gemini $2000 \mathrm{C} / \mathrm{H}(400 \mathrm{MHz})$. Chemical shifts were recorded in $\delta$ (ppm) and the coupling constant $J$ in Hz. Mass spectra were recorded with a JEOL JMS-700. Methyl 5-hydroxynicotinate is available from Tokyo Kasei Kogyo Co. Ltd. (Tokyo).

1.1. Methyl 2-chloro-3-hydroxynicotinate (3)

Methyl 5-hydroxynicotinate (2) $(3.0 \mathrm{mmol})$ was treated dropwise with a sodium hypochlorite solution $(5 \%, 2.1 \mathrm{ml})$ with external chilling on ice. After $30 \mathrm{~min}$ of stirring, the reaction was quenched by adding $4 \mathrm{M} \mathrm{HCl}(10 \mathrm{ml})$. The precipitated crystals were collected by suction, washed with a few milliliters of icecold water, and dried in a vacuum. For analysis, a small crop was sublimated. Mp: $142-143^{\circ} \mathrm{C}$. IR $(\mathrm{KBr}) \mathrm{cm}^{-1}: 3015,1735,1585$ (s), 1240 (vs). ${ }^{1} \mathrm{H}$ NMR $\delta\left(\mathrm{CDCl}_{3}\right): 3.94\left(3 \mathrm{H}, \mathrm{s}, \mathrm{CH}_{3}\right), 5.70(1 \mathrm{H}$, bs, OH), $7.89(1 \mathrm{H}, \mathrm{d}, J=2.3 \mathrm{~Hz}, 4-\mathrm{Py}-\mathrm{H}), 8.59(1 \mathrm{H}, \mathrm{d}, J=2.3 \mathrm{~Hz}$, 6-Py-H); ${ }^{13} \mathrm{C}$ NMR $\delta\left(\mathrm{CDCl}_{3}\right): 52.4,124.1,126.1,140.1,143.2$, 149.5, 152.2. MS m/z (\%): 190 (16), 189 (19), 188 (41), 187 $\left(\mathrm{M}^{+}, 54\right), 186$ (20), 159 (22), 158 (33), 157 (28), 156 (100), 155 (30), 130 (18), 129 (37), 128 (50), 127 (32), 122 (17). Anal. Found: C, 44.80; H, 3.55; N, 7.81\%. Calcd. for $\mathrm{C}_{7} \mathrm{H}_{6} \mathrm{ClNO}_{3}$ : C, $44.82 ; \mathrm{H}, 3.23 ; \mathrm{N}, 7.47 \%$.

1.2. Methyl 3-alkoxy-2-chloronicotinate (4-9)

A mixture of $\mathbf{3}(1.0 \mathrm{mmol})$, the corresponding alkyl iodide $(1.2 \mathrm{mmol})$ and potassium carbonate $(1.5 \mathrm{mmol})$ in acetone $(20 \mathrm{ml})$ was stirred at reflux temperature for $5-8 \mathrm{hr}$. The solvent was removed and the residue was diluted with water. The precipitated solid was washed with chilled water. If the product was a liquid, it was extracted with ethyl acetate and worked up. For analysis, if the product was solid, it was sublimated, and if it was a liquid, a part of it was subjected to preparative thin-layer chromatography with isopropyl ether (IPE)/hexane $1: 2$ to 3 .

1.2.1. Methyl 2-chloro-3-methoxynicotinate (4) Mp: $99-100^{\circ} \mathrm{C}$. IR (KBr) cm ${ }^{-1}: 1730,1430,1390,1250,1215$, 1200, 980, 770, 700. ${ }^{1} \mathrm{H} \mathrm{NMR} \delta\left(\mathrm{CDCl}_{3}\right): 3.97\left(3 \mathrm{H}, \mathrm{s}, \mathrm{OCH}_{3}\right)$, $3.99\left(3 \mathrm{H}, \mathrm{s}, \mathrm{CO}_{2} \mathrm{CH}_{3}\right), 8.58(1 \mathrm{H}, \mathrm{s}, 4-\mathrm{Py}-\mathrm{H}), 8.59(1 \mathrm{H}, \mathrm{s}, 6-\mathrm{Py}-$ $\mathrm{H}) ;{ }^{13} \mathrm{C} \mathrm{NMR} \delta\left(\mathrm{CDCl}_{3}\right): 52.9,56.6,119.3,126.2,141.8,145.2$, 151.7, 165.3. MS m/z (\%): 203 (33), $201\left(\mathrm{M}^{+}, 100\right), 171$ (30), 169 (91), 143 (11), 141 (34), 128 (5), 126 (16). Anal. Found: C, 47.68; H, 3.85; N, 7.02\%. Calcd. for $\mathrm{C}_{8} \mathrm{H}_{8} \mathrm{ClNO}_{3}: \mathrm{C}, 47.66 ; \mathrm{H}$, $4.00 ; \mathrm{N}, 6.95 \%$.

1.2.2. Methyl 2-chloro-3-ethoxynicotinate (5) Mp: $107-108^{\circ} \mathrm{C}$. IR $(\mathrm{KBr}) \mathrm{cm}^{-1}: 1735,1430,1305,1085 .{ }^{1} \mathrm{H}$ NMR $\delta\left(\mathrm{CDCl}_{3}\right): 1.52\left(3 \mathrm{H}, \mathrm{t}, J=7.0 \mathrm{~Hz}, \mathrm{CH}_{2} \underline{\mathrm{C}}_{3}\right), 3.96(3 \mathrm{H}, \mathrm{s}$, 
$\left.\mathrm{CO}_{2} \mathrm{CH}_{3}\right), 4.19\left(2 \mathrm{H}, \quad \mathrm{q}, J=7.0 \mathrm{~Hz}, \mathrm{CH}_{2} \mathrm{CH}_{3}\right), 7.74(1 \mathrm{H}, \mathrm{d}$, $J=1.8 \mathrm{~Hz}, 4-\mathrm{Py}-\mathrm{H}), 8.58(1 \mathrm{H}, \mathrm{d}, J=1.8 \mathrm{~Hz}, 6-\mathrm{Py}-\mathrm{H}) ;{ }^{13} \mathrm{C} \mathrm{NMR} \delta$ $\left(\mathrm{CDCl}_{3}\right): 14.4,52.6,65.2,119.9,125.9,141.4,145.2,150.9$, 165.2. MS $m / z(\%): 217(10), 201\left(\mathrm{M}^{+}, 30\right), 189(21), 187$ (53), 158 (33), 157 (76), 156 (100), 128 (26), 127 (15).

1.2.3. Methyl 2-chloro-3-n-propoxynicotinate (6) Mp: $64^{\circ} \mathrm{C}$. IR (KBr) cm ${ }^{-1}: 1730,1585,1455,1385 .{ }^{1} \mathrm{H}$ NMR $\delta$ $\left(\mathrm{CDCl}_{3}\right): 1.10\left(3 \mathrm{H}, \mathrm{t}, J=7.5 \mathrm{~Hz}, \mathrm{CH}_{2} \mathrm{CH}_{2} \mathrm{CH}_{3}\right), 1.90(2 \mathrm{H}, \mathrm{dt}$, $\left.J=7.5 / 6.5 \mathrm{~Hz}, \mathrm{CH}_{2} \mathrm{CH}_{2} \mathrm{CH}_{3}\right), 3.97\left(3 \mathrm{H}, \mathrm{s}, \mathrm{CO}_{2} \mathrm{CH}_{3}\right), 4.00(2 \mathrm{H}, \mathrm{t}$, $\left.J=6.5 \mathrm{~Hz}, \mathrm{CH}_{2} \mathrm{CH}_{2} \mathrm{CH}_{3}\right), 7.74(1 \mathrm{H}, \mathrm{s}, 4-\mathrm{Py}-\mathrm{H}), 8.57(1 \mathrm{H}, \mathrm{s}, 6-\mathrm{Py}-$ $\mathrm{H}) ;{ }^{13} \mathrm{C} \mathrm{NMR} \delta\left(\mathrm{CDCl}_{3}\right): 10.4,22.2,52.6,71.0,119.9,125.9$, 141.3, 145.3, 151.0, 165.2. MS $m / z$ (\%): 231 (5), $229\left(\mathrm{M}^{+}, 16\right)$, 198 (6), 189 (29), 187 (87), 158 (31), 156 (87), 128 (25), 43 (100).

\subsubsection{Methyl 2-chloro-3-i-propoxynicotinate (7)}

Colorless liquid. IR (liquid) $\mathrm{cm}^{-1}: 1740,1425,1245,1085 .{ }^{1} \mathrm{H}$ NMR $\delta\left(\mathrm{CDCl}_{3}\right): 1.41\left(6 \mathrm{H}, \mathrm{d}, J=5.9 \mathrm{~Hz},\left(\mathrm{CH}_{3}\right)_{2} \mathrm{CH}\right), 3.94(3 \mathrm{H}, \mathrm{s}$, $\left.\mathrm{CO}_{2} \mathrm{CH}_{3}\right), 4.65\left(1 \mathrm{H}\right.$, septet, $\left.J=5.9 \mathrm{~Hz},\left(\mathrm{CH}_{3}\right)_{2} \mathrm{CH}\right), 7.74(1 \mathrm{H}, \mathrm{d}$, $J=1.8 \mathrm{~Hz}, 4-\mathrm{Py}-\mathrm{H}), 8.55(1 \mathrm{H}, \mathrm{d}, J=1.8 \mathrm{~Hz}, 6-\mathrm{Py}-\mathrm{H}) ;{ }^{13} \mathrm{C}$ NMR $\delta$ $\left(\mathrm{CDCl}_{3}\right): 22.0,52.8,72.8,121.9,126.1,141.6,145.5,150.20$, 165.5. MS m/z (\%): $215\left(\mathrm{M}^{+}-15,23\right), 193$ (14), 162 (10), 161 (35), 160 (37), 159 (100), 158 (39), 132 (23), 130 (71), 129 (43), 124 (13). HRMS (EI) $\mathrm{m} / z\left(\mathrm{M}^{+}\right)$: Calcd. for $\mathrm{C}_{10} \mathrm{H}_{12} \mathrm{ClNO}_{3}$ : 229.0506, Found: 229.0520.

1.2.5. Methyl 3-n-butoxy-2-chloro-nicotinate (8)

Pale yellow liquid. IR (liquid) $\mathrm{cm}^{-1}: 1740,1585,1245,1120 .{ }^{1} \mathrm{H}$ NMR $\delta\left(\mathrm{CDCl}_{3}\right): 1.01\left(3 \mathrm{H}, \mathrm{t}, J=7.5 \mathrm{~Hz}, \mathrm{CH}_{2} \mathrm{CH}_{2} \mathrm{CH}_{2} \mathrm{CH}_{3}\right), 1.54$ $\left(2 \mathrm{H}, \mathrm{m}, \mathrm{CH}_{2} \mathrm{CH}_{2} \mathrm{CH}_{2} \mathrm{CH}_{3}\right), 1.86\left(2 \mathrm{H}, \mathrm{m}, \mathrm{CH}_{2} \mathrm{CH}_{2} \mathrm{CH}_{2} \mathrm{CH}_{3}\right), 3.96$ $\left(3 \mathrm{H}, \mathrm{s}, \mathrm{CO}_{2} \mathrm{CH}_{3}\right), 4.12\left(2 \mathrm{H}, \mathrm{t}, J=7.5 \mathrm{~Hz}, \mathrm{CH}_{2} \mathrm{CH}_{2} \mathrm{C}_{2} \mathrm{H}_{5}\right), 7.74$ $(1 \mathrm{H}, \mathrm{d}, J=1.7 \mathrm{~Hz}, 4-\mathrm{Py}-\mathrm{H}), 8.57(1 \mathrm{H}, \mathrm{d}, J=1.7 \mathrm{~Hz}, 6-\mathrm{Py}-\mathrm{H}) ;{ }^{13} \mathrm{C}$ NMR $\delta\left(\mathrm{CDCl}_{3}\right): 13.7,19.1,30.8,52.6,69.3,119.9,125.9$, 141.3, 145.4, 151.1, 165.2. MS $m / z(\%): 245$ (7), $243\left(\mathrm{M}^{+}, 20\right)$, 190 (24), 189 (38), 188 (70), 187 (100), 1585 (28), 156 (80), 128 (23).

1.2.6. Methyl 2-chloro-3-n-pentyloxynicotinate (9)

Pale yellow liquid. IR (liquid) $\mathrm{cm}^{-1}: 1735,1585,1420,1245 .{ }^{1} \mathrm{H}$ NMR $\delta\left(\mathrm{CDCl}_{3}\right): 0.95\left(3 \mathrm{H}, \mathrm{t}, J=6.8 \mathrm{~Hz},\left(\mathrm{CH}_{2}\right)_{4} \mathrm{C}_{3}\right), 1.43(2 \mathrm{H}$, $\left.\mathrm{m},\left(\mathrm{CH}_{2}\right)_{3} \mathrm{CH}_{2} \mathrm{CH}_{3}\right), 1.50\left(2 \mathrm{H}, \mathrm{m}, \mathrm{CH}_{2} \mathrm{CH}_{2} \mathrm{CH}_{2} \mathrm{C}_{2} \mathrm{H}_{5}\right), 1.86(2 \mathrm{H}$, $\left.\mathrm{m}, \mathrm{CH}_{2} \mathrm{CH}_{2} \mathrm{C}_{3} \mathrm{H}_{7}\right), 3.96\left(3 \mathrm{H}, \mathrm{s}, \mathrm{CO}_{2} \mathrm{CH}_{3}\right), 4.12(2 \mathrm{H}, \mathrm{m}$, $\left.\mathrm{C}_{2} \mathrm{C}_{4} \mathrm{H}_{9}\right), 7.74(1 \mathrm{H}, \mathrm{d}, J=2.1 \mathrm{~Hz}, 4-\mathrm{Py}-\mathrm{H}), 8.57(1 \mathrm{H}, \mathrm{d}$, $J=2.1 \mathrm{~Hz}, 6-\mathrm{Py}-\mathrm{H}) ;{ }^{13} \mathrm{C} \mathrm{NMR} \delta\left(\mathrm{CDCl}_{3}\right): 13.9,22.3,27.9,28.4$, $52.8,69.5,119.8,125.8,141.3,145.2,151.0,165.2$. MS $m / z(\%)$ : 259 (31), 257 ( $\left.\mathrm{M}^{+}, 91\right), 226$ (29), 224 (39), 223 (31), 222 (61), 221 (40), 192 (15), 190 (89), 189 (75), 188 (100), 187 (100), 158 (24), 156 (75), $128(21)$.

\subsection{3-Alkoxy-2-chloronicotinic acid (10-14)}

A solution of methyl ester $(1 \mathrm{mmol})$ in methanol $(0.6 \mathrm{ml})$ was treated with $10 \%$ aq. $\mathrm{NaOH}(3 \mathrm{ml})$ and stirred at room temperature for $12 \mathrm{hr}$. IPE washings were discarded and the aqueous phase was acidified to $\mathrm{pH} 2$ with $6 \mathrm{M} \mathrm{HCl}$ with cooling on ice. After the flask was stood for a few hours in an ice bath, the precipitated solid was collected by suction, washed with a few milliliters of chilled water and dried. For analysis, a small crop was sublimated.
1.3.1. 2-Chloro-3-methoxynicotinic acid (10)

Mp: $188-189^{\circ} \mathrm{C}$. IR (KBr) cm ${ }^{-1}$ : 2510, 1725, 1695, 1400, 1285. ${ }^{1} \mathrm{H}$ NMR $\delta$ (acetone- $\left.d_{6}\right): 3.99\left(3 \mathrm{H}, \mathrm{s}, \mathrm{CO}_{2} \mathrm{CH}_{3}\right), 7.83(1 \mathrm{H}, \mathrm{s}, 4-$ Py-H), $8.48(1 \mathrm{H}, \mathrm{s}, 6-\mathrm{Py}-\mathrm{H}) ;{ }^{13} \mathrm{C}-\mathrm{NMR} \delta$ (acetone- $\left.d_{6}\right): 56.7$, 120.1, 127.7, 141.5, 143.8, 151.5, 166.0. MS m/z (\%): 189 (55), 188 (17), $187\left(\mathrm{M}^{+}, 100\right), 170$ (14), 146 (26), 145 (12), 144 (73), 142 (10), 100 (15), 97 (10). Anal. Found: C, 44.54; H, 3.06; N, 7.86\%. Calcd. for $\mathrm{C}_{7} \mathrm{H}_{6} \mathrm{ClNO}_{3}: \mathrm{C}, 44.82 ; \mathrm{H}, 3.23 ; \mathrm{N}, 7.47 \%$.

1.3.2. 2-Chloro-3-ethoxynicotinic acid (11)

Mp: $161-164^{\circ} \mathrm{C}$. IR (KBr) cm ${ }^{-1}: 2500,1715,1585,1420,1385$, 1210. ${ }^{1} \mathrm{H} \mathrm{NMR} \delta\left(\mathrm{CDCl}_{3}\right): 1.34\left(3 \mathrm{H}, \mathrm{t}, J=7.0 \mathrm{~Hz}, \mathrm{CH}_{2} \mathrm{CH}_{3}\right), 4.01$ $\left(2 \mathrm{H}, \mathrm{q}, J=7.0 \mathrm{~Hz}, \mathrm{CH}_{2} \mathrm{CH}_{3}\right), 7.61(1 \mathrm{H}, \mathrm{d}, J=2.0 \mathrm{~Hz}, 4-\mathrm{Py}-\mathrm{H})$, $8.40(1 \mathrm{H}, \mathrm{d}, J=2.0 \mathrm{~Hz}, 6-\mathrm{Py}-\mathrm{H}) ;{ }^{13} \mathrm{C}-\mathrm{NMR} \delta\left(\mathrm{CDCl}_{3}\right): 14.1,64.7$, 120.0, 126.6, 141.3, 144.2, 150.3, 166.1. MS $m / z(\%): 203$ (11), 202 (11), $201\left(\mathrm{M}^{+}, 34\right), 200$ (27), 175 (34), 174 (33), 173 (00), 172 (81), 128 (10), 127 (10), 109 (24), 108 (17).

1.3.3. 2-Chloro-3-i-propoxynicotinic acid (12)

Mp: $140-143^{\circ} \mathrm{C}$. IR ( $\left.\mathrm{KBr}\right) \mathrm{cm}^{-1}: 2500,1715,1580,1420,1285$, 1205. ${ }^{1} \mathrm{H}$ NMR $\delta\left(\mathrm{CDCl}_{3}\right): 1.34\left(6 \mathrm{H}, \mathrm{d}, J=5.9 \mathrm{~Hz}, \mathrm{CH}\left(\mathrm{C}_{3}\right)_{2}\right)$, $4.70\left(2 \mathrm{H}\right.$, septet, $\left.J=5.9 \mathrm{~Hz}, \mathrm{C} \underline{\mathrm{H}}\left(\mathrm{CH}_{3}\right)_{2}\right), 7.83(1 \mathrm{H}, \mathrm{d}, J=1.8 \mathrm{~Hz}$, 4-Py-H), $8.71(1 \mathrm{H}, \mathrm{d}, J=1.8 \mathrm{~Hz}, 6-\mathrm{Py}-\mathrm{H}) ;{ }^{13} \mathrm{C} \mathrm{NMR} \delta\left(\mathrm{CDCl}_{3}\right)$ : 21.7, 72.7, 120.0, 125.2, 141.9, 147.2, 150.2, 169.7. MS $m / z(\%)$ : $215\left(\mathrm{M}^{+}, 6\right), 175$ (34), 174 (10), 173 (100), 109 (9).

1.3.4. 3-n-Butoxy-2-chloronicotinic acid (13)

Mp: $115-116^{\circ} \mathrm{C}$. IR $(\mathrm{KBr}) \mathrm{cm}^{-1}: 2960,1730,1385 .{ }^{1} \mathrm{H}$ NMR $\delta$ $\left(\mathrm{CDCl}_{3}\right): 1.03\left(3 \mathrm{H}, \mathrm{t}, J=7.3 \mathrm{~Hz},\left(\mathrm{CH}_{2}\right)_{3} \mathrm{CH}_{3}\right), 1.57(2 \mathrm{H}, \mathrm{m}$, $\mathrm{CH}_{2} \mathrm{CH}_{2} \mathrm{CH}_{2} \mathrm{CH}_{3}$ ), $1.88\left(2 \mathrm{H}, \mathrm{m}, 2, \mathrm{CH}_{2} \mathrm{CH}_{2} \mathrm{C}_{2} \mathrm{H}_{5}\right), 4.11(2 \mathrm{H}, \mathrm{m}$, $\left.\mathrm{CH}_{2} \mathrm{CH}_{2} \mathrm{C}_{3} \mathrm{H}_{5}\right), 7.94(1 \mathrm{H}, \mathrm{d}, J=1.8 \mathrm{~Hz}, 4-\mathrm{Py}-\mathrm{H}), 8.68(1 \mathrm{H}, \mathrm{d}$, $J=1.8 \mathrm{~Hz}, 6-\mathrm{Py}-\mathrm{H}) ;{ }^{13} \mathrm{C}$ NMR $\delta\left(\mathrm{CDCl}_{3}\right): 13.7,19.1,30.8,69.4$, 120.3, 125.4, 141.9, 146.2, 151.3, 169.6. MS $m / z$ (\%): 231 (5), 229 (M+, 15), 176 (21), 175 (34), 174 (65), 173 (93), 81 (14), 41 (100). HRMS (EI) $\mathrm{m} / z\left(\mathrm{M}^{+}\right)$: Calcd. for $\mathrm{C}_{10} \mathrm{H}_{12} \mathrm{ClNO}_{3}$ : 229.0506, Found: 229.0533.

1.3.5. 2-Chloro-3-n-pentyloxynicotinic acid (14)

Mp: $111-113^{\circ} \mathrm{C} . \mathrm{IR}(\mathrm{KBr}) \mathrm{cm}^{-1}: 2960,1735,1685,1420,1395$, 1210. ${ }^{1} \mathrm{H}$ NMR $\delta\left(\mathrm{CDCl}_{3}\right): 0.95\left(3 \mathrm{H}, \mathrm{t}, J=7.4 \mathrm{~Hz},\left(\mathrm{CH}_{2}\right)_{4} \mathrm{CH}_{3}\right)$, $1.41\left(2 \mathrm{H}, \mathrm{m},\left(\mathrm{CH}_{2}\right)_{3} \mathrm{C}_{2} \mathrm{CH}_{3}\right), 1.51\left(2 \mathrm{H}, \mathrm{m}, \mathrm{CH}_{2} \mathrm{CH}_{2} \mathrm{CH}_{2} \mathrm{C}_{2} \mathrm{H}_{5}\right)$, $1.89\left(2 \mathrm{H}, \quad \mathrm{m}, \quad \mathrm{CH}_{2} \mathrm{CH}_{2} \mathrm{C}_{3} \mathrm{H}_{7}\right), 4.11 \quad(2 \mathrm{H}, \quad \mathrm{t}, \quad J=6.8 \mathrm{~Hz}$, $\left.\mathrm{C}_{2} \mathrm{CH}_{2} \mathrm{C}_{3} \mathrm{H}_{7}\right), 7.79(1 \mathrm{H}, \mathrm{d}, J=1.7 \mathrm{~Hz}, 4-\mathrm{Py}-\mathrm{H}), 8.68(1 \mathrm{H}, \mathrm{d}$, $J=1.7 \mathrm{~Hz}, 6-\mathrm{Py}-\mathrm{H}) ;{ }^{13} \mathrm{C}$ NMR $\delta\left(\mathrm{CDCl}_{3}\right): 14.0,22.3,28.0,28.4$, 69.7, 120.3, 125.3, 141.8, 146.1, 151.2, 169.6. MS m/z (\%): 245 (18), $243\left(\mathrm{M}^{+}, 56\right), 210$ (25), 209 (20), 208 (40), 207 (26), 176 (60), 175 (58), 174 (100), 173 (98), 156 (15).

1.4. 3-Alkoxy-2-chloro-3-pyridinemethanol (6-21)

A suspension of carboxylic acid $(1.0 \mathrm{mmol})$ in chloroform $(5 \mathrm{ml})$ was treated with thionyl chloride $(1 \mathrm{ml})$ and the mixture was warmed at $50^{\circ} \mathrm{C}$ for $5 \mathrm{hr}$. Chloroform and excess thionyl chloride were distilled off in a vacuum, $5 \mathrm{ml}$ of fresh chloroform was added to the flask containing the residue and the distillation was repeated to remove trace amounts of thionyl chloride. The residue was dissolved in a minimal amount of THF ( $c a .1 \mathrm{ml})$ and added dropwise to a previously prepared cold suspension of sodium borohydride $(10 \mathrm{mmol})$ in $6 \mathrm{ml}$ of water with ice cooling. The mixture was stirred for $3 \mathrm{hr}$ in an ice bath. IPE $(15 \mathrm{ml})$ was added to the reaction flask and the entire mixture was stirred for $30 \mathrm{~min}$. 
The IPE layer was separated by decantation, fresh IPE was added to the reaction slurry and stirred, and the IPE layer was again separated by decantation. This separation procedure was repeated once more. The combined IPE extract was washed with water, and dried. After removal of the solvent, the residue was separated by preparative thin-layer chromatography with ethyl acetate as the eluent. The yields of the alcohols were around $60 \%$ based on the carboxylic acids.

1.4.1. 2-Chloro-3-methoxypyridine-5-methanol (16)

Mp: $83-84^{\circ} \mathrm{C}$. IR $(\mathrm{KBr}) \mathrm{cm}^{-1}$ : 3300, 1405, 1085. ${ }^{1} \mathrm{H}$ NMR $\delta$ $\left(\mathrm{CDCl}_{3}\right): 3.91\left(3 \mathrm{H}, \mathrm{s}, \mathrm{CH}_{3}\right), 4.69\left(2 \mathrm{H}, \mathrm{s}, \mathrm{CH}_{2}\right), 7.31(1 \mathrm{H}, \mathrm{s}, 4-\mathrm{Py}-$ $\mathrm{H}), 7.83(1 \mathrm{H}, \mathrm{s}, 6-\mathrm{Py}-\mathrm{H}) ;{ }^{13} \mathrm{C} \mathrm{NMR} \delta\left(\mathrm{CDCl}_{3}\right): 56.0,61.3,116.1$, 118.1, 137.2, 138.0, 139.1, 151.5. MS m/z (\%): 175 (33), 173 $\left(\mathrm{M}^{+}, 100\right), 158$ (18), 133 (22), 131 (56), 106 (26). Anal. Found: C, 48.62; H, 4.36; N, 8.12\%. Calcd. for $\mathrm{C}_{7} \mathrm{H}_{8} \mathrm{ClNO}_{2}: \mathrm{C}, 48.43 ; \mathrm{H}$, $4.64 ; \mathrm{N}, 8.07 \%$.

1.4.2. 2-Chloro-3-ethoxypyridine-5-methanol (17) $\mathrm{Mp}: \quad 60^{\circ} \mathrm{C}$. IR $(\mathrm{KBr}) \mathrm{cm}^{-1}: 3225,1435,1205 .{ }^{1} \mathrm{H}$ NMR $\delta$ $\left(\mathrm{CDCl}_{3}\right): 1.49\left(3 \mathrm{H}, \mathrm{t}, J=7.0 \mathrm{~Hz}, \mathrm{CH}_{3} \mathrm{CH}_{2}\right), 4.12(2 \mathrm{H}, \mathrm{q}$, $\left.J=7.0 \mathrm{~Hz}, \mathrm{CH}_{3} \mathrm{CH}_{2}\right), 4.70\left(2 \mathrm{H}, \mathrm{s}, \mathrm{Py}-\mathrm{CH}_{2}\right), 7.27(1 \mathrm{H}, \mathrm{d}$, $J=1.1 \mathrm{~Hz}, 4-\mathrm{Py}-\mathrm{H}), 7.85$ (1H, d, $J=1.1 \mathrm{~Hz}, 6-\mathrm{Py}-\mathrm{H}) ;{ }^{13} \mathrm{C}$ NMR $\delta$ $\left(\mathrm{CDCl}_{3}\right): 14.6,61.8,65.0,119.1,136.9,138.2,139.9,151.4 . \mathrm{MS}$ $m / z$ (\%): 189 (27), $187\left(\mathrm{M}^{+}, 81\right), 160$ (23), 159 (66), 158 (59), 132 (32), 130 (100), 124 (26), 96 (35).

1.4.3. 2-Chloro-3-i-propoxypyridine-5-methanol (19) $\mathrm{Mp}: 40-41{ }^{\circ} \mathrm{C}$. IR $(\mathrm{KBr}): \mathrm{cm}^{-1} 3310,1415 .{ }^{1} \mathrm{H}$ NMR $\delta\left(\mathrm{CDCl}_{3}\right)$ : $1.40\left(6 \mathrm{H}, \mathrm{d}, J=5.7 \mathrm{~Hz},\left(\mathrm{CH}_{3}\right)_{2} \mathrm{CH}\right), 4.58(1 \mathrm{H}$, septet, $J=5.7 \mathrm{~Hz}$, $\left.\left(\mathrm{CH}_{3}\right)_{2} \mathrm{CH}\right), 4.70\left(2 \mathrm{H}, \mathrm{s}, \mathrm{Py}-\mathrm{CH}_{2}\right), 7.29(1 \mathrm{H}, \mathrm{s}, 4-\mathrm{Py}-\mathrm{H}), 7.87$ $(1 \mathrm{H}, \mathrm{s}, 6-\mathrm{Py}-\mathrm{H}) ;{ }^{13} \mathrm{C} \mathrm{NMR} \delta\left(\mathrm{CDCl}_{3}\right): 21.8,61.5,72.3,121.1$, 137.0, 138.1, 140.6, 150.2. MS m/z (\%): 203 (11), $201\left(\mathrm{M}^{+}, 33\right)$, 161 (36), 160 (36), 159 (100), 158 (34), 132 (25), 130 (79), 96 (22), 94 (24).

1.4.4. 3-n-Butoxy-2-chloropyridine-5-methanol (20)

Colorless liquid. IR (liquid) $\mathrm{cm}^{-1}: 3250,1425,1205,1085 .{ }^{1} \mathrm{H}$ NMR $\delta\left(\mathrm{CDCl}_{3}\right): 1.00\left(3 \mathrm{H}, \mathrm{t}, J=7.4 \mathrm{~Hz},\left(\mathrm{CH}_{2}\right)_{3} \mathrm{CH}_{3}\right), 1.54(2 \mathrm{H}$, $\left.\mathrm{m}, \mathrm{CH}_{2} \mathrm{CH}_{2} \mathrm{CH}_{2} \mathrm{CH}_{3}\right), 1.86\left(2 \mathrm{H}, \mathrm{m}, \mathrm{CH}_{2} \underline{\mathrm{C}}_{2} \mathrm{C}_{2} \mathrm{H}_{5}\right), 4.05(2 \mathrm{H}, \mathrm{t}$, $\left.J=6.6 \mathrm{~Hz}, \mathrm{C}_{2} \mathrm{CH}_{2} \mathrm{C}_{2} \mathrm{H}_{5}\right), 4.71\left(2 \mathrm{H}, \mathrm{s}, \mathrm{Py}_{-} \mathrm{CH}_{2}\right), 7.27(1 \mathrm{H}, \mathrm{d}$, $J=1.7 \mathrm{~Hz}, 4-\mathrm{Py}-\mathrm{H}), 7.89$ (1H, d, $J=1.7 \mathrm{~Hz}, 6-\mathrm{Py}-\mathrm{H}) ;{ }^{13} \mathrm{C}$ NMR $\delta$ $\left(\mathrm{CDCl}_{3}\right): 13.8,19.1,30.9,61.7,69.0,119.0,136.8,138.0,139.9$, 151.3. MS $m / z(\%): 217(14), 215\left(\mathrm{M}^{+}, 43\right), 162$ (11), 161 (47), 160 (43), 159 (100), 158 (30), 149 (11), 132 (21), 130 (66), 124 (21).

1.4.5. 2-Chloro-3-n-pentyloxypyridine-5-methanol (21) Colorless liquid. IR (liquid) $\mathrm{cm}^{-1}: 3305,1420,1085 .{ }^{1} \mathrm{H}$ NMR $\delta$ $\left(\mathrm{CDCl}_{3}\right): 0.94\left(3 \mathrm{H}, \mathrm{t}, J=6.9 \mathrm{~Hz},\left(\mathrm{CH}_{2}\right)_{3} \mathrm{CH}_{2} \mathrm{CH}_{3}\right), 1.40(2 \mathrm{H}, \mathrm{m}$, $\left.\left(\mathrm{CH}_{2}\right)_{3} \mathrm{CH}_{2} \mathrm{CH}_{3}\right), 1.46\left(2 \mathrm{H}, \mathrm{m}, \mathrm{CH}_{2} \mathrm{CH}_{2} \underline{\mathrm{C}}_{2} \mathrm{C}_{2} \mathrm{H}_{5}\right), 1.83(2 \mathrm{H}, \mathrm{m}$, $\left.\mathrm{CH}_{2} \mathrm{CH}_{2} \mathrm{C}_{3} \mathrm{H}_{7}\right), 3.48(1 \mathrm{H}$, bs, $\mathrm{OH}), 4.02(2 \mathrm{H}, \mathrm{t}, J=6.8 \mathrm{~Hz}$, $\left.\mathrm{CH}_{2} \mathrm{CH}_{2} \mathrm{C}_{3} \mathrm{H}_{7}\right), 4.68\left(2 \mathrm{H}, \mathrm{s}, \mathrm{Py}-\mathrm{CH}_{2}\right), 7.26(1 \mathrm{H}, \mathrm{d}, J=1.7 \mathrm{~Hz}, 4-$ Py-H), $7.82(1 \mathrm{H}, \mathrm{d}, J=1.7 \mathrm{~Hz}, 6-\mathrm{Py}-\mathrm{H}) ;{ }^{13} \mathrm{C} \mathrm{NMR} \delta\left(\mathrm{CDCl}_{3}\right)$ : 14.2, 22.6, 28.3, 28.8, 62.1, 69.5, 119.1, 136.8, 138.4, 139.9, 151.5. MS $m / z(\%): 231(10), 229\left(\mathrm{M}^{+}, 29\right), 162$ (10), 161 (22), 160 (34), 159 (64), 158 (12), 145 (40), 144 (42), 143 (100), 142 (93), 130 (26), $116(41), 114$ (98).

1.4.6. 2-Chloro-3-n-propoxy-3-pyridinemethanol (18)

To a suspension of $\mathrm{NaBH}_{4}(3 \mathrm{mmol})$ in $6 \mathrm{ml}$ of THF was added powdered anhydrous $\mathrm{CaCl}_{2}(2 \mathrm{mmol})$. After one-hour of stirring, methyl ester (12) $(1 \mathrm{mmol})$ in $6 \mathrm{ml}$ of THF was added dropwise and the mixture was stirred overnight at room temperature. After diluting in ethyl acetate $(10 \mathrm{ml})$ for $1 \mathrm{hr}$, the upper layer was separated, and again $10 \mathrm{ml}$ of ethyl acetate $(10 \mathrm{ml})$ was added and the separation repeated. Finally brine $(10 \mathrm{ml})$ and ethyl acetate were added and stirred for $10 \mathrm{~min}$ before a similar separation. The combined organic phase was washed with brine $(10 \mathrm{ml})$ and dried. After a work-up, the alcohol was separated. The yield was $10 \%$. Colorless liquid. IR (liquid) $\mathrm{cm}^{-1}: 3350,1410,1380 .{ }^{1} \mathrm{H}$ NMR $\delta\left(\mathrm{CDCl}_{3}\right): 1.06\left(3 \mathrm{H}, \mathrm{t}, J=7.4 \mathrm{~Hz}, \mathrm{CH}_{2} \mathrm{CH}_{2} \mathrm{CH}_{3}\right), 1.86(2 \mathrm{H}$, $\left.\mathrm{dt}, \quad J=6.9 / 7.4 \mathrm{~Hz}, \quad \mathrm{CH}_{2} \mathrm{CH}_{2} \mathrm{CH}_{3}\right), 3.98(2 \mathrm{H}, \quad \mathrm{t}, \quad J=6.9 \mathrm{~Hz}$, $\left.\mathrm{CH}_{2} \mathrm{CH}_{2} \mathrm{CH}_{3}\right), 4.67\left(2 \mathrm{H}, \mathrm{s}, \mathrm{Py}-\mathrm{CH}_{2}\right), 7.25(1 \mathrm{H}, \mathrm{s}, 4-\mathrm{Py}-\mathrm{H}), 7.80$ $(1 \mathrm{H}, \mathrm{s}, 6-\mathrm{Py}-\mathrm{H}) ;{ }^{13} \mathrm{C}-\mathrm{NMR} \delta\left(\mathrm{CDCl}_{3}\right): 10.3,22.2,61.3,70.6$, 119.1, 137.2, 137.8, 139.4, 151.1. MS m/z (\%): 203 (10), 201 $\left(\mathrm{M}^{+}, 29\right), 166$ (10), 164 (17), 161 (33), 160 (23), 159 (100), 158 (45), 132 (26), 130 (82), 129 (26).

1.5. (5-Alkoxy-6-chloro-3-pyridyl)methyl chloride (22-27) A solution of alcohol $(1 \mathrm{mmol})$ in chloroform $(5 \mathrm{ml})$ was treated with $1 \mathrm{ml}$ of thionyl chloride. The solution was warmed at $50^{\circ} \mathrm{C}$ for $3 \mathrm{hr}$. The cooled solution was poured into ice-cold water $(15 \mathrm{ml})$ with stirring. After $1 \mathrm{hr}$ of stirring, the chloroform layer was separated and the aqueous layer was extracted with chloroform $(5 \mathrm{ml} \times 2)$. The combined chloroform layer was washed with water, $1 \%$ aq. $\mathrm{NaHCO}_{3}$, and brine, and dried. After removal of the solvent, the residue was subjected to preparative thin-layer chromatography with IPE/Hexane $1: 2$. The yields of the products were around $70 \%$.

1.5.1. (6-Chloro-5-methoxypyridin-3-yl)methyl chloride (22) Mp: $30-31{ }^{\circ} \mathrm{C}$. IR (liquid) $\mathrm{cm}^{-1}: 1405,1305 .{ }^{1} \mathrm{H} \mathrm{NMR} \delta\left(\mathrm{CDCl}_{3}\right)$ : $3.95\left(3 \mathrm{H}, \mathrm{s}, \mathrm{CH}_{3}\right), 4.58\left(2 \mathrm{H}, \mathrm{s}, \mathrm{CH}_{2}\right), 7.26(1 \mathrm{H}, \mathrm{d}, J=1.5 \mathrm{~Hz}, 4-$ Py-H), 7.97 (1H, d, $J=1.5 \mathrm{~Hz}, 6-\mathrm{Py}-\mathrm{H}) ;{ }^{13} \mathrm{C} \mathrm{NMR} \delta\left(\mathrm{CDCl}_{3}\right)$ : 42.3, 56.1, 119.0, 133.3, 139.4, 140.7, 151.5. MS m/z (\%): 193 (47), $191\left(\mathrm{M}^{+}, 72\right), 158$ (57), 156 (100), 106 (18). HRMS (EI) $\mathrm{m} / z\left(\mathrm{M}^{+}\right)$: Calcd. for $\mathrm{C}_{7} \mathrm{H}_{7} \mathrm{Cl}_{2} \mathrm{NO}$ : 190.9906, Found: 190.9912 .

1.5.2. (6-Chloro-5-ethoxypyridin-3-yl)methyl chloride (23) $\mathrm{Mp}: 7^{\circ} \mathrm{C}$. IR $(\mathrm{KBr}) \mathrm{cm}^{-1}: 1420,1385,1310 .{ }^{1} \mathrm{H}$ NMR $\delta$ $\left(\mathrm{CDCl}_{3}\right): 1.51\left(3 \mathrm{H}, \mathrm{t}, J=7.4 \mathrm{~Hz}, \mathrm{CH}_{2} \mathrm{CH}_{3}\right), 4.16(2 \mathrm{H}, \mathrm{q}$, $\left.J=7.4 \mathrm{~Hz}, \mathrm{CH}_{2} \mathrm{CH}_{3}\right), 4.56\left(2 \mathrm{H}, \mathrm{s}, \mathrm{CH}_{2} \mathrm{Cl}\right), 7.23(1 \mathrm{H}, \mathrm{s}, 4-\mathrm{Py}-\mathrm{H})$, $7.96(1 \mathrm{H}, \mathrm{s}, 6-\mathrm{Py}-\mathrm{H}) ;{ }^{13} \mathrm{C} \mathrm{NMR} \delta\left(\mathrm{CDCl}_{3}\right): 14.6,42.5,65.1$, 120.1, 133.3, 139.4, 141.2, 151.2. MS $m / z$ (\%): 207 (31), 205 $\left(\mathrm{M}^{+}, 47\right), 179$ (40), 177 (63), 170 (18), 144 (51), 142 (100).

1.5.3. (6-Chloro-5-n-propoxypyridin-3-yl)methyl chloride (24) Colorless liquid. IR (liquid) $\mathrm{cm}^{-1}: 1420,1390,1310 .{ }^{1} \mathrm{H}$ NMR $\delta$ $\left(\mathrm{CDCl}_{3}\right): 1.09\left(3 \mathrm{H}, \mathrm{t}, J=7.4 \mathrm{~Hz}, \mathrm{CH}_{2} \mathrm{CH}_{2} \underline{\mathrm{H}}_{3}\right), 1.89(2 \mathrm{H}, \mathrm{dt}$, $\left.J=6.9 / 7.4 \mathrm{~Hz}, \mathrm{CH}_{2} \mathrm{CH}_{2} \mathrm{CH}_{3}\right), 4.03\left(2 \mathrm{H}, \mathrm{t}, J=6.9 \mathrm{~Hz}, \mathrm{C}_{2} \mathrm{C}_{2} \mathrm{H}_{5}\right)$, $4.56\left(2 \mathrm{H}, \mathrm{s}, \mathrm{CH}_{2} \mathrm{Cl}\right), 7.23(1 \mathrm{H}, \mathrm{s}, 4-\mathrm{Py}-\mathrm{H}), 7.95(1 \mathrm{H}, \mathrm{s}, 6-\mathrm{Py}-\mathrm{H})$; ${ }^{13} \mathrm{C}$ NMR $\delta\left(\mathrm{CDCl}_{3}\right): 10.3,22.2,42.4,70.7,120.0,133.2,139.2$, 141.1, 151.1. MS m/z (\%): 221 (10), $219\left(\mathrm{M}^{+}, 15\right), 179$ (45), 177 (76), 176 (38), 144 (28), 142 (89).

1.5.4. (6-Chloro-5-i-propoxypyridin-3-yl)methylchloride (25) $\mathrm{Mp}: 51^{\circ} \mathrm{C}$. IR $(\mathrm{KBr}) \mathrm{cm}^{-1}: 1420,1385,1300 .{ }^{1} \mathrm{H} \mathrm{NMR} \delta$ $\left(\mathrm{CDCl}_{3}\right): 1.41\left(6 \mathrm{H}, \mathrm{d}, J=6.3 \mathrm{~Hz},\left(\mathrm{CH}_{3}\right)_{2} \mathrm{CH}\right), 4.56(2 \mathrm{H}, \mathrm{s}$, $\left.\mathrm{CH}_{2} \mathrm{Cl}\right), 4.60\left(1 \mathrm{H}\right.$, septet, $\left.J=6.3 \mathrm{~Hz},\left(\mathrm{CH}_{3}\right)_{2} \mathrm{CH}\right), 7.25(1 \mathrm{H}, \mathrm{d}$, $J=1.8 \mathrm{~Hz}, 4-\mathrm{Py}-\mathrm{H}), 7.96(1 \mathrm{H}, \mathrm{d}, J=1.8 \mathrm{~Hz}, 6-\mathrm{Py}-\mathrm{H}) ;{ }^{13} \mathrm{C}$ NMR $\delta$ 
$\left(\mathrm{CDCl}_{3}\right): 21.8,42.4,72.5,122.0,133.2,139.5,142.3,150.2 . \mathrm{MS}$ m/z (\%): $221\left(\mathrm{M}^{+}, 13\right), 219$ (6), $217\left(\mathrm{M}^{+}, 9\right), 183$ (11), 182 (100), 180 (11), 146 (11), 140 (34), 137 (12).

1.5.5. (5-n-Butoxy-6-chloropyridin-3-yl)methyl chloride (26) Pale yellow liquid. IR (liquid) $\mathrm{cm}^{-1}: 1420,1385,1310 .{ }^{1} \mathrm{H}$ NMR $\delta \quad\left(\mathrm{CDCl}_{3}\right): 1.00 \quad\left(3 \mathrm{H}, \quad \mathrm{m}, \quad\left(\mathrm{CH}_{2}\right)_{3} \mathrm{CH}_{3}\right), 1.53 \quad(2 \mathrm{H}, \quad \mathrm{m}$, $\left.\mathrm{CH}_{2} \mathrm{CH}_{2} \mathrm{CH}_{2} \mathrm{CH}_{3}\right), 1.82\left(2 \mathrm{H}, \mathrm{m}, \mathrm{CH}_{2} \mathrm{C}_{2} \mathrm{C}_{2} \mathrm{H}_{5}\right), 4.05(2 \mathrm{H}, \mathrm{t}$, $\left.J=6.6 \mathrm{~Hz}, \mathrm{CH}_{2} \mathrm{CH}_{2} \mathrm{C}_{2} \mathrm{H}_{5}\right), 4.55\left(2 \mathrm{H}, \mathrm{s}, \mathrm{CH}_{2} \mathrm{Cl}\right), 7.22(1 \mathrm{H}, \mathrm{d}$, $J=1.8 \mathrm{~Hz}, 4-\mathrm{Py}-\mathrm{H}), 7.94(1 \mathrm{H}, \mathrm{d}, J=1.8 \mathrm{~Hz}, 6-\mathrm{Py}-\mathrm{H}) ;{ }^{13} \mathrm{C}$ NMR $\delta$ $\left(\mathrm{CDCl}_{3}\right): 13.8,19.2,31.0,42.5,69.2,120.0,133.3,139.3,141.3$, 151.4. MS m/z (\%): 235 (11), $233\left(\mathrm{M}^{+}, 18\right), 200$ (8), 198 (33), 143 (32), 141 (100), 106 (8).

1.5.6. (6-Chloro-5-n-pentyloxypyridine-3-yl)methyl chloride (27) Pale yellow liquid. IR (liquid) $\mathrm{cm}^{-1}: 1425,1385,1310 .{ }^{1} \mathrm{H}$ NMR $\delta\left(\mathrm{CDCl}_{3}\right): 0.95\left(3 \mathrm{H}, \mathrm{t}, J=7.4 \mathrm{~Hz},\left(\mathrm{CH}_{2}\right)_{3} \mathrm{CH}_{2} \mathrm{CH}_{3}\right), 1.42(2 \mathrm{H}, \mathrm{m}$, $\left.\left(\mathrm{CH}_{2}\right)_{3} \mathrm{CH}_{2} \mathrm{CH}_{3}\right), 1.49\left(2 \mathrm{H}, \mathrm{m}, \mathrm{CH}_{2} \mathrm{CH}_{2} \mathrm{CH}_{2} \mathrm{C}_{2} \mathrm{H}_{5}\right), 1.87(2 \mathrm{H}, \mathrm{m}$, $\left.\mathrm{CH}_{2} \mathrm{CH}_{2} \mathrm{CH}_{2} \mathrm{C}_{2} \mathrm{H}_{5}\right), 4.06\left(2 \mathrm{H}, \mathrm{t}, J=6.6 \mathrm{~Hz}, \mathrm{CH}_{2} \mathrm{CH}_{2} \mathrm{C}_{3} \mathrm{H}_{7}\right), 4.56$ $\left(2 \mathrm{H}, \mathrm{s}, \mathrm{CH}_{2} \mathrm{Cl}\right), 7.23(1 \mathrm{H}, \mathrm{d}, J=1.2 \mathrm{~Hz}, 4-\mathrm{Py}-\mathrm{H}), 7.95(1 \mathrm{H}, \mathrm{d}$, $J=1.2 \mathrm{~Hz}, 6-\mathrm{Py}-\mathrm{H}) ;{ }^{13} \mathrm{C} \mathrm{NMR} \delta\left(\mathrm{CDCl}_{3}\right): 13.9,22.3,28.0,28.5$, 42.4, 69.3, 119.9, 133.2, 139.2, 141.1, 151.2. MS $m / z$ (\%): 249 (34), 247 (M+ , 52), 212 (23), 180 (45), 179 (69), 177 (100).

1.6. 1-(5-Alkoxy-6-chloro-3-pyridylmethyl)-2-nitroiminoimidazolidines (1d-1i)

The preparation of $\mathbf{1 d}$ is typical of the procedure used. A solution of 2-nitroimino-4-imidazoline ${ }^{3)}(\mathbf{2 8} ; 214 \mathrm{mg}, 1.65 \mathrm{mmol})$ in DMF $(15 \mathrm{ml})$ was treated with sodium hydride $(60 \%$ oil dispersion, $72 \mathrm{mg}, 1.80 \mathrm{mmol})$ below $5^{\circ} \mathrm{C}$. The mixture was stirred at room temperature until no hydrogen evolved and then cooled again to $0^{\circ} \mathrm{C}$. A solution of $22(315 \mathrm{mg}, 1.66 \mathrm{mmol})$ in DMF $(5 \mathrm{ml})$ was added dropwise. The cooling bath was set aside and the solution was stirred at room temperature for $3.5 \mathrm{hr}$. The reaction was quenched with one drop of acetic acid and the DMF was evaporated in vacuum. Chromatography on $\mathrm{SiO}_{2}$ with ethyl acetate gave $256 \mathrm{mg}$ (54\% yield) of product, which was purified by recrystallization from methanol. Mp: $185-188^{\circ} \mathrm{C}$. IR $(\mathrm{KBr}) \mathrm{cm}^{-1}$ : $3300,1600-1520,1400,1250,1200 .{ }^{1} \mathrm{H}$ NMR $\delta$ (DMSO- $d_{6}$ ): $3.48\left(2 \mathrm{H}, \mathrm{m}, \mathrm{NCH}_{2} \mathrm{CH}_{2} \mathrm{~N}\right), 3.80\left(2 \mathrm{H}, \mathrm{m}, \mathrm{NCH}_{2} \mathrm{CH}_{2} \mathrm{~N}\right), 3.91(3 \mathrm{H}$, s, $\left.\mathrm{CH}_{3}\right), 4.48\left(2 \mathrm{H}, \mathrm{s}, \mathrm{CH}_{2}\right), 7.52(1 \mathrm{H}, \mathrm{d}, J=1.8 \mathrm{~Hz}, 4-\mathrm{Py}-\mathrm{H}), 7.93$ $(1 \mathrm{H}, \mathrm{d}, J=1.8 \mathrm{~Hz}, 6-\mathrm{Py}-\mathrm{H}), 9.00(1 \mathrm{H}, \mathrm{bs}, \mathrm{NH}) ;{ }^{13} \mathrm{C} \mathrm{NMR}$ $\left(\mathrm{DMSO}-d_{6}\right)$ : 41.8, 45.0, 45.4, 56.5, 120.4, 133.1, 138.5, 139.5, 151.3, 160.6. MS m/z (\%): 288 (4), $286\left(\mathrm{M}^{+}, 11\right), 241$ (36), 236 (100), 224 (14), 204 (22), 203 (24), 189 (11), 156 (9). Anal. Found: C, 42.32; H, 4.19; N, 24.63\%. Calcd. for $\mathrm{C}_{10} \mathrm{H}_{12} \mathrm{ClN}_{5} \mathrm{O}_{3}$ : C, 42.04; H, 4.24; N, 24.52\%.

1.6.1. 1-(6-Chloro-5-ethoxy-3-pyridylmethyl)-2-nitroiminoimidazolidine (1e)

Mp: $150-151^{\circ} \mathrm{C}$. IR (KBr) cm ${ }^{-1}: 3180,1580-1520,1470,1455$, 1420. ${ }^{1} \mathrm{H}$ NMR $\delta$ (DMSO- $\left.d_{6}\right): 1.49\left(3 \mathrm{H}, \mathrm{t}, J=7.0 \mathrm{~Hz}, \mathrm{CH}_{2} \underline{\mathrm{C}}_{3}\right)$, $3.54\left(2 \mathrm{H}, \mathrm{m}, \mathrm{NCH}_{2} \mathrm{CH}_{2} \mathrm{~N}\right), 3.80\left(2 \mathrm{H}, \mathrm{m}, \mathrm{NCH}_{2} \mathrm{CH}_{2} \mathrm{~N}\right), 4.12(2 \mathrm{H}$, q, J=7.0 Hz, $\left.\underline{\mathrm{H}}_{2} \mathrm{CH}_{3}\right), 4.51\left(2 \mathrm{H}, \mathrm{s}, \mathrm{Py}-\mathrm{CH}_{2}\right), 7.27(1 \mathrm{H}, \mathrm{d}$, $J=1.5 \mathrm{~Hz}, 4-\mathrm{Py}-\mathrm{H}), 7.87(1 \mathrm{H}, \mathrm{d}, J=1.5 \mathrm{~Hz}, 6-\mathrm{Py}-\mathrm{H}), 8.19(1 \mathrm{H}$, bs, $\mathrm{NH}) ;{ }^{13} \mathrm{C}$ NMR $\delta$ (DMSO- $\left.d_{6}\right): 14.4,41.5,45.2,45.54,65.1$, 120.1, 130.8, 139.4, 141.2, 151.4, 161.2. MS $m / z(\%): 300\left(\mathrm{M}^{+}\right.$, 14), 256 (23), 255 (35), 255 (35), 253 (100), 225 (38), 218 (24), 217 (23), 189 (39), 161 (16), 142 (15). Anal. Found: C, 44.12; H,
4.59; N, 23.83\%. Calcd. for $\mathrm{C}_{11} \mathrm{H}_{14} \mathrm{ClN}_{5} \mathrm{O}_{3}: \mathrm{C}, 44.08 ; \mathrm{H}, 4.71 ; \mathrm{N}$, $23.37 \%$.

1.6.2. 1-(6-Chloro-5-n-propoxy-3-pyridylmethyl)-2-nitroiminoimidazolidine (1f)

$\mathrm{Mp}: \quad 125^{\circ} \mathrm{C}$. IR $(\mathrm{KBr}) \mathrm{cm}^{-1}$ : 3370, 1600-1540, 1455-1420 1230-1200. ${ }^{1} \mathrm{H}$ NMR $\delta\left(\mathrm{CDCl}_{3}\right): 1.08(3 \mathrm{H}, \mathrm{t}, J=7.4 \mathrm{~Hz}$, $\left.\mathrm{CH}_{2} \mathrm{CH}_{2} \mathrm{CH}_{3}\right), 1.85\left(2 \mathrm{H}, \mathrm{dt}, J=7.4 / 6.3 \mathrm{~Hz}, \mathrm{CH}_{2} \underline{\mathrm{C}}_{2} \mathrm{CH}_{3}\right), 3.54$ $\left(2 \mathrm{H}, \mathrm{m}, \mathrm{NCH}_{2} \mathrm{CH}_{2} \mathrm{~N}\right), 3.82\left(2 \mathrm{H}, \mathrm{m}, \mathrm{NCH}_{2} \mathrm{CH}_{2} \mathrm{~N}\right), 4.02(2 \mathrm{H}, \mathrm{q}$, $\left.J=6.3 \mathrm{~Hz}, \mathrm{CH}_{2} \mathrm{CH}_{2} \mathrm{CH}_{3}\right), 4.51\left(2 \mathrm{H}, \mathrm{s}, \mathrm{Py}_{-} \mathrm{CH}_{2}\right), 7.26(1 \mathrm{H}, \mathrm{d}$, $J=1.7 \mathrm{~Hz}, 4-\mathrm{Py}-\mathrm{H}), 7.86(1 \mathrm{H}, \mathrm{d}, J=1.7 \mathrm{~Hz}, 6-\mathrm{Py}-\mathrm{H}), 8.22(1 \mathrm{H}$, bs, $\mathrm{NH}) ;{ }^{13} \mathrm{C}$ NMR $\delta\left(\mathrm{CDCl}_{3}\right): 10.3,22.2,41.5,45.2,45.5,70.8$, 120.1, 130.8, 139.3, 141.1, 151.4, 161.2. MS m/z (\%): $314\left(\mathrm{M}^{+}\right.$, 10), 270 (25), 269 (35), 268 (16), 267 (100), 232 (16), 231 (15), 227 (16), 225 (46), 190 (29). Anal. Found: C, 46.12; H, 5.11; N, $22.03 \%$. Calcd. for $\mathrm{C}_{12} \mathrm{H}_{16} \mathrm{ClN}_{5} \mathrm{O}_{3}: \mathrm{C}, 45.94 ; \mathrm{H}, 5.14 ; \mathrm{N}, 22.32 \%$.

1.6.3. 1-(6-Chloro-5-i-propoxy-3-pyridylmethyl)-2-nitroiminoimidazolidine (1g)

Mp: $126^{\circ} \mathrm{C}$. IR (KBr) cm ${ }^{-1}: 3335,1600-1540,1455-1420,1205$. ${ }^{1} \mathrm{H}$ NMR $\delta\left(\mathrm{CDCl}_{3}\right): 1.39\left(6 \mathrm{H}, \mathrm{d}, J=6.3 \mathrm{~Hz},\left(\mathrm{CH}_{3}\right)_{2} \mathrm{CH}\right), 3.54$ $\left(2 \mathrm{H}, \mathrm{m}, \mathrm{NCH}_{2} \mathrm{CH}_{2} \mathrm{~N}\right), 3.81\left(2 \mathrm{H}, \mathrm{m}, \mathrm{NCH}_{2} \mathrm{CH}_{2} \mathrm{~N}\right), 4.51(2 \mathrm{H}, \mathrm{s}$, $\left.\mathrm{CH}_{2}\right), 4.59\left(1 \mathrm{H}\right.$, septet, $\left.J=6.3 \mathrm{~Hz},\left(\mathrm{CH}_{3}\right)_{2} \mathrm{C} \underline{\mathrm{H}}\right), 7.31(1 \mathrm{H}, \mathrm{d}$, $J=1.7 \mathrm{~Hz}, 4-\mathrm{Py}-\mathrm{H}), 7.86(1 \mathrm{H}, \mathrm{d}, J=1.7 \mathrm{~Hz}, 6-\mathrm{Py}-\mathrm{H}), 8.19(1 \mathrm{H}$, bs, $\mathrm{NH}) ;{ }^{13} \mathrm{C}$ NMR $\delta\left(\mathrm{CDCl}_{3}\right): 21.7,41.5,45.2,45.5,72.5,122.1$, 130.7, 139.5, 142.3, 150.5, 161.2. MS $m / z(\%): 312\left(\mathrm{M}^{+}, 1\right), 269$ (10), 267 (31), 266 (100), 232 (12), 231 (12), 226 (85), 224 (100), 197 (18), 190 (55), 189 (96), 162 (38), 161 (73). Anal. Found: C, 45.82; H, 5.21; N, 21.99\%. Calcd. for $\mathrm{C}_{12} \mathrm{H}_{16} \mathrm{ClN}_{5} \mathrm{O}_{3}$ : C, 45.94; H, 5.14; N, 22.32\%.

1.6.4. 1-(5-n-Butoxy-6-chloro-3-pyridylmethyl)-2-nitroiminoimidazolidine $\mathbf{( 1 \mathbf { h } )}$

Mp: $126-127^{\circ} \mathrm{C}$. IR (KBr) cm ${ }^{-1}: 3375$ (m), 2960 (m), 15901560 (vs), 1480 (m), 1435-1420 (vs), 1285 (vs), 1240 (vs), 1145 (m), 1205 (s), 1080 (m), 945 (m), $750(\mathrm{~m}) .{ }^{1} \mathrm{H}-\mathrm{NMR} \delta\left(\mathrm{CDCl}_{3}\right)$ : $0.99\left(3 \mathrm{H}, \mathrm{m},\left(\mathrm{CH}_{2}\right)_{3} \mathrm{CH}_{3}\right), 1.52\left(2 \mathrm{H}, \mathrm{m},\left(\mathrm{CH}_{2}\right)_{2} \mathrm{CH}_{2} \mathrm{CH}_{3}\right), 1.85$ $\left(2 \mathrm{H}, \mathrm{m}, \mathrm{CH}_{2} \mathrm{CH}_{2} \mathrm{C}_{2} \mathrm{H}_{5}\right), 3.55\left(2 \mathrm{H}, \mathrm{m}, \mathrm{NCH}_{2} \mathrm{CH}_{2} \mathrm{~N}\right), 3.81(2 \mathrm{H}, \mathrm{m}$, $\left.\mathrm{NCH}_{2} \mathrm{CH}_{2} \mathrm{~N}\right), 4.05\left(2 \mathrm{H}, \mathrm{m}, \mathrm{CH}_{2} \mathrm{CH}_{2} \mathrm{C}_{2} \mathrm{H}_{5}\right), 4.51\left(2 \mathrm{H}, \mathrm{s}, \mathrm{Py}-\mathrm{CH}_{2}\right)$, $7.31(1 \mathrm{H}, \mathrm{d}, J=1.6 \mathrm{~Hz}, 4-\mathrm{Py}-\mathrm{H}), 7.86(1 \mathrm{H}, \mathrm{d}, J=1.6 \mathrm{~Hz}, 6-\mathrm{Py}-\mathrm{H})$, $8.22(1 \mathrm{H}, \mathrm{bs}, \mathrm{NH}) ;{ }^{13} \mathrm{C}-\mathrm{NMR} \delta\left(\mathrm{CDCl}_{3}\right): 13.7,19.0,30.8,41.5$, $45.2,45.5,69.1,120.0,130.8,139.2,141.1,151.4,161.1$. MS $\mathrm{m} / \mathrm{z}(\%): 328\left(\mathrm{M}^{+}+1,26\right), 283$ (36), $282(19), 281$ (100), 246 (13), 245 (13), 227 (15), 225 (46), 189 (20), 161 (10). Anal. Found: C, 47.22; H, 5.41; N, 21.50\%. Calcd. for $\mathrm{C}_{13} \mathrm{H}_{18} \mathrm{ClN}_{5} \mathrm{O}_{3}$ : C, 47.64; H, 5.53; N, 21.37\%.

1.6.5. 1-(6-Chloro-5-n-pentyloxy-3-pyridylmethyl)-2-nitroiminoimidazolidine (1i)

Mp: $127-128^{\circ} \mathrm{C}$. IR (KBr) cm ${ }^{-1}$ : 3320, 1585, 1425, 1310, 1200, 1080. ${ }^{1} \mathrm{H} \quad \mathrm{NMR} \quad \delta \quad\left(\mathrm{CDCl}_{3}\right): 0.94 \quad(3 \mathrm{H}, \quad \mathrm{t}, \quad J=7.4 \mathrm{~Hz}$, $\left.\left(\mathrm{CH}_{2}\right)_{3} \mathrm{CH}_{2} \mathrm{CH}_{3}\right), 1.40\left(2 \mathrm{H}, \mathrm{m},\left(\mathrm{CH}_{2}\right)_{3} \mathrm{C}_{2} \mathrm{CH}_{3}\right), 1.47(2 \mathrm{H}, \mathrm{m}$, $\left.\mathrm{CH}_{2} \mathrm{CH}_{2} \mathrm{CH}_{2} \mathrm{CH}_{2} \mathrm{CH}_{3}\right), 1.85\left(2 \mathrm{H}, \mathrm{m}, \mathrm{CH}_{2} \mathrm{CH}_{2} \mathrm{C}_{3} \mathrm{H}_{7}\right), 3.55(2 \mathrm{H}, \mathrm{m}$, $\left.\mathrm{NCH}_{2} \mathrm{CH}_{2} \mathrm{~N}\right), 3.81\left(2 \mathrm{H}, \mathrm{m}, \mathrm{NCH}_{2} \mathrm{CH}_{2} \mathrm{~N}\right), 4.03(2 \mathrm{H}, \mathrm{t}, J=6.3 \mathrm{~Hz}$, $\left.\mathrm{CH}_{2} \mathrm{CH}_{2} \mathrm{C}_{3} \mathrm{H}_{7}\right), 4.51\left(2 \mathrm{H}, \mathrm{s}, \mathrm{Py}-\mathrm{CH}_{2}\right), 7.25(1 \mathrm{H}, \mathrm{d}, J=1.8 \mathrm{~Hz}, 4-$ Py-H), 7.86 (1H, d, $J=1.8 \mathrm{~Hz}, 6-\mathrm{Py}-\mathrm{H}), 8.22(1 \mathrm{H}, \mathrm{bs}, \mathrm{NH}) ;{ }^{13} \mathrm{C}$ NMR $\delta\left(\mathrm{CDCl}_{3}\right): 13.9,22.2,28.0,28.5,41.5,45.2,45.5,69.4$, 120.0, 130.8, 139.2, 141.1, 151.54, 161.1. MS $m / z(\%): 341\left(\mathrm{M}^{+}\right.$, 3), 297 (32), 296 (12), 295 (100), 260 (9), 228 (18), 227 (36), 
225 (45), 189 (13), 161 (14). Anal. Found: C, 49.32; H, 5.84; N, $20.65 \%$. Calcd. for $\mathrm{C}_{14} \mathrm{H}_{20} \mathrm{ClN}_{5} \mathrm{O}_{3}: \mathrm{C}, 49.19 ; \mathrm{H}, 5.90 ; \mathrm{N}, 20.49 \%$.

\section{Insecticidal tests}

Insecticidal tests were conducted as described earlier. ${ }^{1)}$ The minimum lethal dose (MLD; mol) was determined for each test compound. The activity was expressed as $\log (1 / \mathrm{MLD})$. Results are listed in Table 1. Each value is the mean of at least two experimental runs. The standard error was within \pm 0.2 .

\section{Results and Discussion}

\section{Synthesis}

Figure 2 outlines the preparation of 5-alkoxyimidacloprid derivatives. A commercially available 5-hydroxynicotinic ester (2) was chlorinated at the 2-position with a sodium hypochlorite solution at $0^{\circ} \mathrm{C}$. First, we attempted the direct reduction of ester to the alcohol using 5-propoxy ester (6). The reduction with $\mathrm{NaBH}_{4}$ in refluxing methanol, with $\mathrm{LiAlH}_{4}$ in ether at lower temperatures, with diisobutylaluminum hydride in toluene and with boranemethyl sulfide complex failed. Only the method using $\mathrm{NaBH}_{4}$ in the presence of $\mathrm{CaCl}_{2}$ afforded alcohol $\mathbf{1 7}$ in as a low yield as $10 \%$. Therefore, we adopted a detour approach, reduction of acid chloride (15), and obtained the other alcohols in satisfactory yields. The final products $(\mathbf{1 d}-\mathbf{1 i})$ were obtained by a coupling reaction of (5-alkoxy-6-chloropyridin-3-yl)methyl chloride (22-27) with 2-nitroiminoimidazolidine (28) in DMF using sodium hydride as an acid acceptor. The spectral data supported the structures of six new products.
Table 1. Insecticidal activity of compounds tested ${ }^{a}$

\begin{tabular}{llrrr}
\hline & & \multicolumn{3}{c}{$\log (1 / \mathrm{MLD})(\mathrm{mol})$} \\
No. & \multicolumn{1}{c}{$\mathrm{X}$} & Alone & $+\mathrm{NIA}$ & $+(\mathrm{PB}+\mathrm{NIA})$ \\
\hline $\mathbf{1 a}^{b)}$ & $\mathrm{H}$ & 8.96 & 10.15 & 10.15 \\
$\mathbf{1 b}^{b)}$ & $\mathrm{F}$ & 8.82 & 9.91 & 9.91 \\
$\mathbf{1 c}^{b)}$ & $\mathrm{Cl}$ & 8.35 & 9.05 & 9.05 \\
$\mathbf{1 d}$ & $\mathrm{OMe}$ & 7.43 & 8.74 & 8.74 \\
$\mathbf{1 e}$ & $\mathrm{OEt}$ & 7.07 & 7.87 & 8.08 \\
$\mathbf{1 f}$ & $\mathrm{OPr}(n)$ & $<6.89$ & 6.89 & 6.89 \\
$\mathbf{1 g}$ & $\mathrm{OPr}(i)$ & 6.99 & 7.89 & 8.10 \\
$\mathbf{1 h}$ & $\mathrm{OBu}(n)$ & 7.12 & 8.12 & 8.12 \\
$\mathbf{1 i}$ & $\mathrm{OPent}(n)$ & 5.86 & 6.46 & 6.46 \\
\hline
\end{tabular}

${ }^{a)}$ See Fig. 1 for general structure. ${ }^{b)}$ Ref. 1.

\section{Insecticidal activity}

The insecticidal activity $24 \mathrm{hr}$ after injection into American cockroaches is listed in Table 1. Without a synergist (alone), the minimum lethal dose (log 1/MLD, mol) of imidacloprid (1a) was in the nanomolar range. The derivatives were considerably less potent than imidacloprid. Among the new compounds, the methoxy derivative (1d) was most active with a $\log (1 / \mathrm{MLD})$ value of 7.43 . The potency is 35 times smaller than that of imidacloprid, and about 10 times larger than that of the fluoro and chloro derivatives $(\mathbf{1 b}, \mathbf{1 c})$ at the molar level. The introduction of higher<smiles>COC(=O)c1cnc(Cl)c(O)c1</smiles>

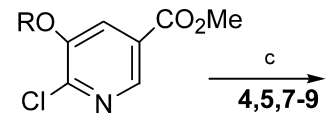

$4(\mathrm{R}=\mathrm{Me}), \mathbf{5}(\mathrm{R}=\mathrm{Et})$ $6(\mathrm{R}=n-\mathrm{Pr}), 7(\mathrm{R}=i-\mathrm{Pr})$ $8(\mathrm{R}=n-\mathrm{Bu}), \boldsymbol{9}(\mathrm{R}=n-\mathrm{Pentyl})$<smiles>[R6]Oc1cc(C(=O)O)cnc1Cl</smiles><smiles>CC(C)Cc1cnc(Cl)c(O)c1</smiles><smiles>COc1cc(C(=O)Cl)cnc1Cl</smiles>

16-21

$24(\mathrm{R}=n-\mathrm{Pr}), 25(\mathrm{R}=i-\mathrm{Pr})$<smiles>[R20]c1cc(CCl)cnc1Cl</smiles>

$26(\mathrm{R}=n-\mathrm{Bu}), 27(\mathrm{R}=n-\mathrm{Pentyl})$<smiles>[R6]c1cc(CCl)cnc1Cl</smiles>

22-27

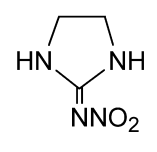

28<smiles>[R]Oc1cc(CN2CCNC2[N+](=O)[O-])cnc1Cl</smiles>

$1 d-1 i$

Fig. 2. Synthesis of 5-alkoxyimidacloprid derivatives. Reagents and conditions: (a) $\mathrm{NaOCl}, 0^{\circ} \mathrm{C}$; (b) $\mathrm{RI}, \mathrm{K}_{2} \mathrm{CO}_{3}$, acetone, reflux, 5-8 hr; (c) $10 \% \mathrm{NaOH}, \mathrm{MeOH}$, room temp, $12 \mathrm{hr}$; (d) (i) $\mathrm{SOCl}_{2}, 50^{\circ} \mathrm{C}, 5 \mathrm{hr}$ (ii) $\mathrm{NaBH}_{4}, \mathrm{H}_{2} \mathrm{O} / \mathrm{THF}, 0^{\circ} \mathrm{C}, 3 \mathrm{hr}$; (e) $\mathrm{NaBH}_{4}, \mathrm{CaCl}_{2}, \mathrm{THF}$, room temp, $12 \mathrm{hr}$; (f) $\mathrm{SOCl}_{2}, \mathrm{CHCl}_{3}, 5 \mathrm{hr} ;(\mathrm{g}) \mathrm{NaH} / \mathrm{DMF}, 3-4 \mathrm{hr}$. 
alkoxy homologues did not improve the activity further. NIA16388 (propargyl propyl benzenephosphonate; NIA), which was originally reported as an inhibitor of the hydrolytic metabolism of tetramethrin, ${ }^{4)}$ a pyrethroid, has also been used to improve the activity of neonicotinoids. Our previous experiments suggested that the synergistic effect of NIA was ascribed to the inhibition of oxidative metabolism at the methylenyl carbon(s) on the imidazolidine ring. ${ }^{5-7)}$ Recent experiments with houseflies revealed a retarded degradation of $\left[{ }^{3} \mathrm{H}\right]$ imidacloprid in the presence of NIA. ${ }^{8)}$ The present results support such an effect: NIA increased the level of activity by about one log unit for each compound. We have reported that the activity of neonicotinoid compounds could be further enhanced by adding $\mathrm{PB}$, another inhibitor of oxidative metabolism. ${ }^{9}$ ) A slight synergistic effect was observed for the OEt and $\mathrm{OPr}^{i}$ derivatives $(\mathbf{1 e}, \mathbf{1 g})$. Under these conditions, the potency of compound $\mathbf{1 d}$ was raised to almost the nanomolar level. The present experiments with and without synergists showed that the relation between the $\log (1 / \mathrm{MLD})$ values of alkoxy derivatives and the alkyl chain lengths was not straightforward, but overall, the longer the alkyl chain, the weaker the activity. This result suggests that the permissible space around the position in question on the pyridine ring is small.

In conclusion, given that the methoxy derivative, the most active compound in the alkoxy series, was inferior to halo-substituted compounds, the extra alkoxy group at the fifth position is unfavorable.

\section{Acknowledgments}

This work was partly supported by a Grant-in-aid for Scientific Research (C) to SK (No. 17580094) from the Japan Society for the Promotion of Science. We thank Earth Chemical Co., Ltd. for donating the American cockroaches.

\section{References}

1) S. Kagabu, N. Ito, R. Imai, Y. Hieta and K. Nishimura: J. Pestic. Sci. 30, 409-413 (2005).

2) S. Kagabu: Rev. Toxicol. 1, 75-129 (1997).

3) P. Tronche, A. Amelot, J. Bayard and C. Laroussinie: Ann. Pharm. Franc. 18, 726-735 (1960).

4) T. Suzuki and J. Miyamoto: Pestic. Biochem. Physiol. 4, 86-97 (1974).

5) K. Nishimura, Y. Kanda, A. Okazawa and T. Ueno: Pestic. Biochem. Physiol. 50, 51-59 (1994).

6) K. Kiriyama, S. Kagabu and K. Nishimura: J. Pestic. Sci. 29, 43-45 (2004).

7) K. Nishimura, M. Tanaka, K. Iwaya and S. Kagabu: Pestic. Biochem. Physiol. 62, 172-178 (1998).

8) H. Nishiwaki, K. Sato, Y. Nakagawa, M. Miyashita and H. Miyagawa: J. Pestic. Sci. 29, 110-116 (2004).

9) S. Kagabu, A. Azuma and K. Nishimura: J. Pestic. Sci. 27, 267271 (2002). 